\title{
Erratum: Competition along trajectories governs adaptation rates towards antimicrobial
} resistance

C. Brandon Ogbunugafor and Margaret J. Eppstein

Nature Ecology \& Evolution 1, 0007 (2016); published 21 November 2016; corrected 9 January 2017.

In the original version of the Supplementary Information for this Article, Supplementary Tables 4 and 5 were misnumbered. The Supplementary Information PDF has been updated accordingly. 